PAPER • OPEN ACCESS

Measurement of the integrated Luminosities of cross-section scan data samples around the $\psi(3770)$ mass region

To cite this article: M. Ablikim et al 2018 Chinese Phys. C 42063001

View the article online for updates and enhancements.
Related content - Measurement of e+e DD cross sections M. Ablikim, M. N. Achasov, S. Ahmed et al.

- Luminosity measurements for the $R$ scan experiment at BESIII

M. Ablikim, M. N. Achasov, S. Ahmed et al.

- Measurements of the center-of-mass energies at BESIII via the di-muon process

M. Ablikim, M. N. Achasov, X. C. Ai et al. 


\section{Measurement of the integrated Luminosities of cross-section scan data samples around the $\psi(3770)$ mass region ${ }^{*}$}

M. Ablikim(麦迪娜) ${ }^{1}$ M. N. Achasov ${ }^{9, d}$ S. Ahmed ${ }^{14}$ M. Albrecht ${ }^{4}$ M. Alekseev ${ }^{55 A, 55 C}$ A. Amoroso ${ }^{55 A, 55 C}$ F. F. An $(\text { 安芬芬 })^{1}$ Q. An (安琪 $)^{42,52}$ Y. Bai(白羽 $)^{41}$ O. Bakina ${ }^{26}$ R. Baldini Ferroli ${ }^{22 A}$ Y. Ban(班勇 $)^{34}$ K. Begzsuren ${ }^{24}$ D. W. Bennett ${ }^{21}$ J. V. Bennett ${ }^{5}$ N. Berger ${ }^{25}$ M. Bertani ${ }^{22 A}$ D. Bettoni ${ }^{23 A}$ F. Bianchi ${ }^{55 A, 55 C}$ E. Boger ${ }^{26, b}$ I. Boyko $^{26}$ R. A. Briere ${ }^{5}$ H. Cai (蔡浩 $)^{57}$ X. Cai (蔡啸 $)^{1,42}$ O. Cakir ${ }^{45 A}$ A. Calcaterra ${ }^{22 A}$ G. F. Cao(曹国富 $)^{1,46}$ S. A. Cetin ${ }^{45 B}$ J. Chai ${ }^{55 C}$ J. F. Chang(常劲帆) $)^{1,42}$ W. L. Chang ${ }^{1,46}$ G. Chelkov ${ }^{26, b, c}$ G. Chen(陈刚) ${ }^{1}$ H. S. Chen(陈和生) $)^{1,46}$ J. C. Chen $(\text { 陈江川 })^{1}$ M. L. Chen $(\text { 陈玛丽 })^{1,42}$ P. L. Chen (陈平亮 $)^{53}$ S. J. Chen (陈申见 $)^{32}$ X. R. Chen (陈旭荣 $)^{29}$ Y. B. Chen (陈元柏 $)^{1,42}$ X. K. Chu(禇新坤 $)^{34}$ G. Cibinetto ${ }^{23 A}$ F. Cossio ${ }^{55 C}$ H. L. Dai(代洪亮 $)^{1,42}$ J. P. Dai(代建平) $)^{37, h}$ A. Dbeyssi ${ }^{14}$ D. Dedovich ${ }^{26}$ Z. Y. Deng(邓子艳) ${ }^{1}$ A. Denig ${ }^{25}$ I. Denysenko ${ }^{26}$ M. Destefanis ${ }^{55 A, 55 C}$ F. De Mori ${ }^{55 A, 55 C}$ Y. Ding $(\text { 丁勇 })^{30}$ C. Dong $(\text { 董超 })^{33}$ J. Dong $(\text { 董静 })^{1,42}$ L. Y. Dong $(\text { 董燎原 })^{1,46}$ M. Y. Dong $(\text { 董明义 })^{1}$ Z. L. Dou(豆正否 $)^{32}$ S. X. Du(杜书先 $)^{60}$ P. F. Duan( $(\mathbf{E} \text { 鹏飞 })^{1}$ J. Fang(方建 $)^{1,42}$ S. S. Fang(房双世) $)^{1,46}$ Y. Fang $(\text { 方易 })^{1}$ R. Farinelli2 ${ }^{23 A, 23 B}$ L. Fava ${ }^{55 B, 55 C}$ S. Fegan ${ }^{25}$ F. Feldbauer ${ }^{4}$ G. Felici ${ }^{22 A}$ C. Q. Feng(封常青) ${ }^{42,52}$ E. Fioravanti ${ }^{23 A}$ M. Fritsch ${ }^{4}$ C. D. Fu(傅 成栋 $)^{1}$ Q. Gao(高清 $)^{1}$ X. L. Gao(高金丕 $)^{42,52}$ Y. Gao(高原宁 $)^{44}$ Y. G. Gao(高勇贵 $)^{6}$ Z. Gao(高榛 $)^{42,52}$ B. Garillon ${ }^{25}$ I. Garzia ${ }^{23 A}$ A. Gilman ${ }^{49}$ K. Goetzen ${ }^{10}$ L. Gong(龚丽) ${ }^{33}$ W. X. Gong(龚文煊 $)^{1,42}$ W. Gradl ${ }^{25}$ M. Greco ${ }^{55 A, 55 C}$ L. M. Gu(谷立民) ${ }^{32}$ M. H. Gu(顾旻皓) ${ }^{1,42}$ Y. T. Gu(顾运厅 $)^{12}$ A. Q. Guo(郭爱强) ${ }^{1}$ L. B. Guo(郭立波 $)^{31}$ R. P. Guo(郭 如盼 $)^{1,46}$ Y. P. Guo(郭玉萍) ${ }^{25}$ A. Guskov ${ }^{26}$ Z. $\operatorname{Haddadi}^{28}$ S. Han(韩爽) ${ }^{57}$ X. Q. Hao(郝喜庆) ${ }^{15}$ F. A. Harris ${ }^{47}$ K. L. He(何康林 $)^{1,46}$ X. Q. He(何希勤 $)^{51}$ F. H. Heinsius ${ }^{4}$ T. Held ${ }^{4}$ Y. K. Heng(衡月昆 $)^{1}$ T. Holtmann ${ }^{4}$ Z. L. Hou(侯治 龙 $)^{1}$ H. M. Hu(胡海明 $)^{1,46}$ J. F. Hu(胡继峰 $)^{37, h}$ T. Hu(胡涛 $)^{1}$ Y. Hu(胡誉 $)^{1}$ G. S. Huang(黄光顺 $)^{42,52}$ J. S. Huang(黄 金书) $)^{15}$ X. T. Huang(黄性涛 $)^{36}$ X. Z. Huang(黄晓忠) ${ }^{32}$ Z. L. Huang(黄智玲 ${ }^{30}$ T. Hussain ${ }^{54}$ W. Ikegami Andersson ${ }^{56}$ M, Irshad ${ }^{42,52}$ Q. Ji(纪全 $)^{1}$ Q. P. Ji(姬清平 $)^{15}$ X. B. Ji(季晓斌 $)^{1,46}$ X. L. Ji(季篠 璐 $)^{1,42}$ X. S. Jiang(江晓山 $)^{1}$ X. Y. Jiang(蒋兴雨 $)^{33}$ J. B. Jiao (焦健斌 $)^{36}$ Z. Jiao(焦铮 $)^{17}$ D. P. Jin $(\text { 金大鹏 })^{1}$ S. Jin $(\text { 金山 })^{1,46}$ Y. Jin $(\text { 金毅 })^{48}$ T. Johansson ${ }^{56}$ A. Julin ${ }^{49}$ N. Kalantar-Nayestanaki ${ }^{28}$ X. S. Kang(康晓珅 $)^{33}$ M. Kavatsyuk ${ }^{28}$ B. C. Ke $(\text { 柯百谦 })^{1}$ T. Khan ${ }^{42,52}$ A. Khoukaz ${ }^{50}$ P. Kiese $^{25}$ R. Kliemt ${ }^{10}$ L. Koch $^{27}$ O. B. Kolcu ${ }^{45 B, f}$ B. Kopf ${ }^{4}$ M. Kornicer ${ }^{47}$ M. Kuemmel ${ }^{4}$ M. Kuessner ${ }^{4}$ A. Kupsc ${ }^{56}$ M. Kurth ${ }^{1}$ W. Kühn ${ }^{27}$ J. S. Lange ${ }^{27}$ M. Lara $^{21}$ P. Larin ${ }^{14}$ L. Lavezzi ${ }^{55 C, 1}$ S. Leiber $^{4}$ H. Leithoff ${ }^{25}$ C. $\operatorname{Li}(\text { 李翠 })^{56}$ Cheng $\operatorname{Li}(\text { 李澄 })^{42,52}$ D. M. Li (李德民 $)^{60}$ F. Li (李飞) $)^{1,42} \mathrm{~F} . \mathrm{Y} . \operatorname{Li}(\text { 李峰云 })^{34} \mathrm{G} \cdot \operatorname{Li}(\text { 李刚 })^{1}$ H. B. $\operatorname{Li}(\text { 李海波 })^{1,46}$ H. J. $\operatorname{Li}(\text { 李惠静 })^{1,46}$ J. C. $\operatorname{Li}(\text { 李家才 })^{1}$ J. W. $\operatorname{Li}(\text { 李井文 })^{40}$ K. J. Li (李凯杰 $)^{43} \operatorname{Kang} \operatorname{Li}($ 李

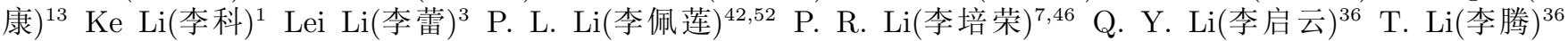
W. D. $\operatorname{Li}(\text { 李卫东 })^{1,46}$ W. G. $\operatorname{Li}(\text { 李卫国 })^{1}$ X. L. Li $(\text { 李晓玲 })^{36}$ X. N. $\operatorname{Li}(\text { 李小男 })^{1,42}$ X. Q. Li (李学潜 $)^{33}$ Z. B. $\operatorname{Li}($ 李 志兵 $)^{43}$ H. Liang(梁吴 $)^{42,52}$ Y. F. Liang(梁勇飞 $)^{39}$ Y. T. Liang(梁羽铁 $)^{27}$ G. R. Liao(廖广睿 $)^{11}$ L. Z. Liao(廖龙 洲 $)^{1,46}$ J. Libby ${ }^{20}$ C. X. Lin(林创新 $)^{43}$ D. X. Lin(林德旭 $)^{14}$ B. Liu(刘冰 $)^{37, h}$ B. J. Liu(刘北江 $)^{1}$ C. X. Liu(刘春 秀 $)^{1}$ D. Liu(刘栋) ${ }^{42,52}$ D. Y. Liu(刘殿宇) ${ }^{37, h}$ F. H. Liu(刘福虎 $)^{38}$ Fang Liu(刘芳) $)^{1}$ Feng Liu(刘峰 $)^{6}$ H. B. Liu(刘 宏邦) $)^{12}$ H. L Liu(刘恒君 $)^{41}$ H. M. Liu(刘怀民 $)^{1,46}$ Huanhuan Liu(刘欢欢 $)^{1}$ Huihui Liu(刘汇慧 $)^{16}$ J. B. Liu(刘建

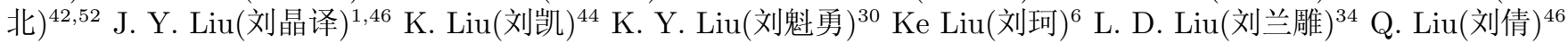
S. B. Liu (刘树涁 $)^{42,52}$ X. Liu(刘翔 $)^{29}$ Y. B. Liu(刘玉斌 $)^{33}$ Z. A. Liu(刘振安) ${ }^{1}$ Zhiqing Liu(刘智青 $)^{25}$ Y. F. Long(龙云 飞 $)^{34}$ X. C. Lou (娄辛丑) $)^{1}$ H. J. Lu(吕海江 $)^{17}$ J. G. Lu(吕军光 $)^{1,42}$ Y. $\mathrm{Lu}(\text { 卢宇 })^{1}$ Y. P. Lu(卢云鹏 $)^{1,42}$ C. L. Luo(罗成 林 $)^{31}$ M. X. Luo(罗民兴 $)^{59}$ X. L. Luo(罗小兰 $)^{1,42}$ S. Lusso ${ }^{55 C}$ X. R. Lyu(吕晓睿) ${ }^{46}$ F. C. Ma (马风才 $)^{30} \mathrm{H} . \mathrm{L} . \mathrm{Ma}($ 马 海龙 $)^{1}$ L. L. Ma (马连良 $)^{36}$ M. M. Ma(马明明 $)^{1,46}$ Q. M. Ma (马秋梅 $)^{1}$ X. N. Ma(马旭宁 $)^{33}$ X. Y. Ma (马骁妍 $)^{1,42}$

Received 13 March 2018, Published online 11 May 2018

* Supported by National Key Basic Research Program of China (2015CB856700), National Natural Science Foundation of China (NSFC) (11235011, 11335008, 11425524, 11625523, 11635010), the Chinese Academy of Sciences (CAS) Large-Scale Scientific Facility Program, the CAS Center for Excellence in Particle Physics (CCEPP), Joint Large-Scale Scientific Facility Funds of the NSFC and CAS (U1332201, U1532257, U1532258), CAS Key Research Program of Frontier Sciences (QYZDJ-SSW-SLH003, QYZDJ-SSW-SLH040), 100 Talents Program of CAS, National 1000 Talents Program of China, INPAC and Shanghai Key Laboratory for Particle Physics and Cosmology, German Research Foundation DFG under Contracts Nos. Collaborative Research Center CRC 1044, FOR 2359, Istituto Nazionale di Fisica Nucleare, Italy, Koninklijke Nederlandse Akademie van Wetenschappen (KNAW) (530-4CDP03), Ministry of Development of Turkey (DPT2006K-120470), National Science and Technology fund, The Swedish Research Council, U. S. Department of Energy (DEFG02-05ER41374, DE-SC-0010118, DE-SC-0010504, DE-SC-0012069), University of Groningen (RuG) and the Helmholtzzentrum fuer Schwerionenforschung GmbH (GSI), Darmstadt, WCU Program of National Research Foundation of Korea (R32-2008-000-10155-0)

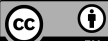

Content from this work may be used under the terms of the Creative Commons Attribution 3.0 licence. Any further distribution of this work must maintain attribution to the author(s) and the title of the work, journal citation and DOI. Article funded by SCOAP ${ }^{3}$ and published under licence by Chinese Physical Society and the Institute of High Energy Physics of the Chinese Academy of Sciences and the Institute of Modern Physics of the Chinese Academy of Sciences and IOP Publishing Ltd 
Y. M. Ma(马玉明 $)^{36}$ F. E. Maas ${ }^{14}$ M. Maggiora ${ }^{55 A, 55 C}$ Q. A. Malik ${ }^{54}$ A. Mangoni ${ }^{2 B}$ Y. J. Mao(冒亚军) ${ }^{34}$ Z. P. Mao(毛 泽普 $)^{1}$ S. Marcello ${ }^{55 A, 55 C}$ Z. X. Meng(孟召霞 $)^{48}$ J. G. Messchendorp ${ }^{28}$ G. Mezzadri ${ }^{23 B}$ J. Min (闵建 $)^{1,42}$ T. J. Min(闵 天觉 $)^{1}$ R. E. Mitchell ${ }^{21}$ X. H. Mo(莫晓虎 $)^{1}$ Y. J. Mo(莫玉俊 $)^{6}$ C. Morales Morales ${ }^{14}$ G. Morello ${ }^{22 A}$ N. Yu. Muchnoi ${ }^{9, d}$ H. Muramatsu ${ }^{49}$ A. Mustafa ${ }^{4}$ S. Nakhoul ${ }^{10, g}$ Y. Nefedov $^{26}$ F. Nerling ${ }^{10}$ I. B. Nikolaev ${ }^{9, d}$ Z. Ning(宁哲 $)^{1,42}$ S. Nisar ${ }^{8}$ S. L. Niu(牛顺利 $)^{1,42}$ X. Y. Niu(牛讯伊 $)^{1,46}$ S. L. Olsen(馬鵬) $)^{35, j}$ Q. Ouyang(欧阳群) $)^{1}$ S. Pacetti ${ }^{22 B}$ Y. Pan(潘 越 $)^{42,52}$ M. Papenbrock ${ }^{56}$ P. Patteri ${ }^{22 A}$ M. Pelizaeus ${ }^{4}$ J. Pellegrino ${ }^{55 A, 55 C}$ H. P. Peng(彭海平 $)^{42,52}$ Z. Y. Peng(彭志 远 $)^{12}$ K. Peters ${ }^{10, g}$ J. Pettersson ${ }^{56}$ J. L. Ping(平加伦) ${ }^{31}$ R. G. Ping(平荣刚) ${ }^{1,46}$ A. Pitka ${ }^{4}$ R. Poling ${ }^{49}$ V. Prasad ${ }^{42,52}$ H. R. Qi (漆红荣 $)^{2}$ M. Qi (祁鸣 $)^{32}$ T. Y. Qi (齐天钰 $)^{2}$ S. Qian(钱森 $)^{1,42}$ C. F. Qiao (乔从丰 $)^{46}$ N. Qin $(\text { 覃拈 })^{57}$ X. S. Qin ${ }^{4}$ Z. H. Qin(秦中华 $)^{1,42}$ J. F. Qiu(邱进发 $)^{1}$ K. H. Rashidd ${ }^{54, i}$ C. F. Redmer ${ }^{25}$ M. Richter ${ }^{4}$ M. Ripka $^{25}$ M. Rolo ${ }^{55 C}$ G. Rong(荣刚) ${ }^{1,46}$ Ch. Rosner ${ }^{14}$ X. D. Ruan(阮向东) ${ }^{12}$ A. Sarantsev ${ }^{26, e}$ M. Savriée ${ }^{23 B}$ C. Schnier ${ }^{4}$ K. Schoenning ${ }^{56}$ W. Shan (单藏) ${ }^{18}$ X. Y. Shan(单心钜 $)^{42,52}$ M. Shao(郡明) ${ }^{42,52}$ C. P. Shen(沈成平) ${ }^{2}$ P. X. Shen(沈培 迅 $)^{33}$ X. Y. Shen (沈肖雁 $)^{1,46}$ H. Y. Sheng(盛华义 ${ }^{1}$ X. Shi(史欣) $)^{1,42}$ J. J. Song(宋娇娇) ${ }^{36}$ W. M. Song ${ }^{36}$ X. Y. Song(宋 欣颖) ${ }^{1}$ S. Sosio ${ }^{55 A, 55 C}$ C. Sowa ${ }^{4}$ S. Spataro ${ }^{55 A, 55 C}$ G. X. Sun(孙功星) ${ }^{1}$ J. F. Sun(孙俊峰) ${ }^{15}$ L. Sun (孙亮 $)^{57}$ S. S. Sun (孙 胜森 $)^{1,46}$ X. H. Sun(孙新华 $)^{1}$ Y. J. Sun(孙勇杰) $)^{42,52}$ Y. K Sun(孙艳坤 $)^{42,52}$ Y. Z. Sun(孙永昭) ${ }^{1}$ Z. J. Sun(孙志嘉 $)^{1,42}$ Z. T. Sun(孙振田 $)^{21}$ Y. T Tan(谭雅星 $)^{42,52}$ C. J. Tang(唐昌建 $)^{39}$ G. Y. Tang(唐光毅 $)^{1}$ X. Tang(唐晓) ${ }^{1}$ I. Tapan $^{45 C}$ M. Tiemens ${ }^{28}$ B. Tsednee ${ }^{24}$ I. Uman ${ }^{45 D}$ G. S. Varner ${ }^{47}$ B. Wang(王斌) ${ }^{1}$ B. L. Wang(王滨龙) ${ }^{46}$ C. W. Wang(王 成伟 $)^{32}$ D. Wang (王东 $)^{34}$ D. Y. Wang (王大勇 $)^{34}$ Dan Wang(王丹 $)^{46}$ K. Wang(王科 $)^{1,42}$ L. L. Wang $(\text { 王亮亮 })^{1}$ L. S. Wang (王灵淑 $)^{1}$ M. Wang (王萌 $)^{36}$ Meng Wang (王蒙 $)^{1,46}$ P. Wang(王平 $)^{1}$ P. L. Wang(王佩良 $)^{1}$ W. P. Wang(王 维平 $)^{42,52}$ X. F. Wang (王雄飞) ${ }^{1}$ Y. Wang (王越 $)^{42,52}$ Y. F. Wang (王贻芳 $)^{1}$ Y. Q. Wang (王亚乾 $)^{25}$ Z. Wang(王 铮 $)^{1,42}$ Z. G. Wang(王志刚 $)^{1,42}$ Z. Y. Wang(王至勇 $)^{1}$ Zongyuan Wang(王宗源 $)^{1,46}$ T. Weber ${ }^{4}$ D. H. Wei(魏代 会 $)^{11}$ P. Weidenkaff ${ }^{25}$ S. P. Wen (文硕频 $)^{1}$ U. Wiedner ${ }^{4}$ M. Wolke ${ }^{56}$ L. H. Wu(伍灵慧 $)^{1}$ L. J. Wu $(\text { 吴连近 })^{1,46}$ Z. $\mathrm{Wu}(\text { 吴智 })^{1,42} \mathrm{~L} . \mathrm{Xia}(\text { 夏否 })^{42,52}$ X. Xia ${ }^{36} \mathrm{Y}$. Xia(夏宇) ${ }^{19}$ D. Xiao(肖栋) ${ }^{1}$ Y. J. Xiao(肖言佳) ${ }^{1,46}$ Z. J. Xiao(肖 振军 $)^{31}$ Y. G. Xie(谢宇广 $)^{1,42}$ Y. H. Xie (谢跃红 $)^{6}$ X. A. Xiong(熊习安 $)^{1,46}$ Q. L. Xiu(修青磊 $)^{1,42}$ G. F. Xu(许国 发 $)^{1} \mathrm{~J} . \mathrm{J} . \mathrm{Xu}(\text { 徐静静 })^{1,46} \mathrm{~L} . \mathrm{Xu}(\text { 徐雷 })^{1}$ Q. J. Xu(徐庆君 $)^{13}$ Q. N. Xu(徐庆年 $)^{46} \mathrm{X} . \mathrm{P} . \mathrm{Xu}(\text { 徐新平 })^{40} \mathrm{~F} . \mathrm{Yan}($ 严 芳 $)^{53}$ L. Yan $(\text { 严亮 })^{55 A, 55 C}$ W. B. Yan(齿文标 $)^{42,52}$ W. C. Yan(间文成 $)^{2}$ Y. H. Yan(颜永红) $)^{19}$ H. J. Yang(杨海军 $)^{37, h}$ H. X. Yang (杨洪勋 $)^{1}$ L. Yang (杨柳 $)^{57}$ S. L. Yang(杨双莉 $)^{1,46}$ Y. H. Yang(杨友华 $)^{32}$ Y. X. Yang(杨永栩 $)^{11}$ Yifan Yang (杨翊凡 $)^{1,46}$ M. Ye(叶梅 $)^{1,42}$ M. H. Ye(叶铭汉 $)^{7}$ J. H. Yin(殷俊吴 $)^{1}$ Z. Y. You(尤郑昀 $)^{43}$ B. X. Yu(俞伯 祥 $)^{1}$ C. X. Yu(喻纯旭) ${ }^{33}$ J. S. Yu(俞洁晟 $)^{29}$ C. Z. Yuan(苑长征 $)^{1,46}$ Y. Yuan(袁野) ${ }^{1}$ A. Yuncu ${ }^{45 B, a}$ A. A. Zafar ${ }^{54}$ A. Zallo ${ }^{22 A}$ Y. Zeng(曾云 $)^{19}$ Z. Zeng(曾哲 $)^{42,52}$ B. X. Zhang(张丙新 $)^{1}$ B. Y. Zhang(张炳云 $)^{1,42}$ C. C. Zhang $(\text { 张长春 })^{1}$ D. H. Zhang(张达华 $)^{1}$ H. H. Zhang(张宏浩 $)^{43}$ H. Y. Zhang(章红宇) $)^{1,42}$ J. Zhang(张晋) $)^{1,46}$ J. L. Zhang(张杰否石 $)^{58}$ J. Q. Zhang ${ }^{4}$ J. W. Zhang(张家文) $)^{1}$ J. Y. Zhang(张建勇) $)^{1}$ J. Z. Zhang(张景芝) ${ }^{1,46}$ K. Zhang(张坤 $)^{1,46}$ L. Zhang(张 否 $)^{44}$ S. F. Zhang(张思凡 ${ }^{32}$ T. J. Zhang(张天骄 $)^{37, h}$ X. Y. Zhang(张学尧) ${ }^{36}$ Y. Zhang(张言 $)^{42,52}$ Y. H. Zhang(张 银鸿 $)^{1,42}$ Y. T. Zhang(张亚腾 $)^{42,52}$ Yang Zhang(张洋 $)^{1}$ Yao Zhang(张瑶) ${ }^{1}$ Yu Zhang(张宇 $)^{46}$ Z. H. Zhang(张正 好 $)^{6}$ Z. P. Zhang(张子平) ${ }^{52}$ Z. Y. Zhang(张振宇) $)^{57}$ G. Zhao(赵光) ${ }^{1}$ J. W. Zhao(赵京伟) $)^{1,42}$ J. Y. Zhao(赵静宜) $)^{1,46}$ J. Z. Zhao(赵京周) $)^{1,42}$ Lei Zhao(赵雷) ${ }^{42,52}$ Ling Zhao(赵玲) ${ }^{1}$ M. G. Zhao(赵明刚) ${ }^{33}$ Q. Zhao(赵强) $)^{1}$ S. J. Zhao(赵 书俊 ${ }^{60}$ T. C. Zhao(赵天池 $)^{1}$ Y. B. Zhao(赵豫斌 $)^{1,42}$ Z. G. Zhao(赵政国) ${ }^{42,52}$ A. Zhemchugov ${ }^{26, b}$ B. Zheng(郑波 $)^{53}$ J. P. Zheng(郑建平) ${ }^{1,42}$ W. J. Zheng(郑文静 $)^{36}$ Y. H. Zheng(郑阳恒 $)^{46}$ B. Zhong(钟涁 $)^{31}$ L. Zhou(周莉) ${ }^{1,42}$ Q. Zhou(周 巧 $)^{1,46}$ X. Zhou(周详 $)^{57}$ X. K. Zhou(周晓康 $)^{42,52}$ X. R. Zhou(周小蓉 $)^{42,52}$ X. Y. Zhou(周兴玉 $)^{1}$ A. N. Zhu(朱傲男) $)^{1,46}$ J. Zhu (朱江) ${ }^{33}$ J. Zhu (朱江) $)^{43}$ K. Zhu(朱凯 $)^{1}$ K. J. Zhu(朱科军 $)^{1}$ S. Zhu(朱帅 $)^{1}$ S. H. Zhu(朱世海 $)^{51}$ X. L. Zhu(朱 相雷 $)^{44}$ Y. C. Zhu(朱莹春 $)^{42,52}$ Y. S. Zhu(朱永生 $)^{1,46}$ Z. A. Zhu(朱自安 $)^{1,46}$ J. Zhuang(庄建 $)^{1,42}$ B. S. Zou(邹冰松 $)^{1}$ J. H. Zou(邹佳恒 $)^{1}$

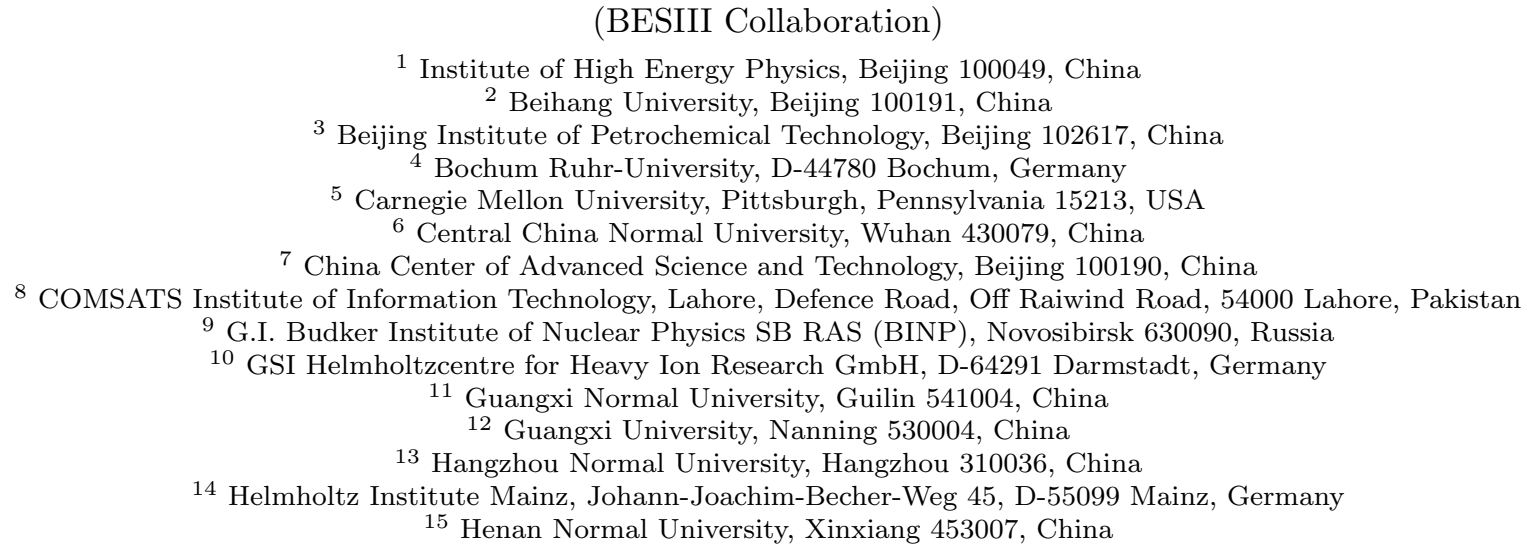


16 Henan University of Science and Technology, Luoyang 471003, China

17 Huangshan College, Huangshan 245000, China

18 Hunan Normal University, Changsha 410081, China

19 Hunan University, Changsha 410082, China

${ }^{20}$ Indian Institute of Technology Madras, Chennai 600036, India

${ }^{21}$ Indiana University, Bloomington, Indiana 47405, USA

22 (A)INFN Laboratori Nazionali di Frascati, I-00044, Frascati, Italy; (B)INFN and University of Perugia, I-06100, Perugia, Italy

23 (A)INFN Sezione di Ferrara, I-44122, Ferrara, Italy; (B)University of Ferrara, I-44122, Ferrara, Italy

24 Institute of Physics and Technology, Peace Ave. 54B, Ulaanbaatar 13330, Mongolia

25 Johannes Gutenberg University of Mainz, Johann-Joachim-Becher-Weg 45, D-55099 Mainz, Germany

${ }^{26}$ Joint Institute for Nuclear Research, 141980 Dubna, Moscow region, Russia

27 Justus-Liebig-Universitaet Giessen, II. Physikalisches Institut, Heinrich-Buff-Ring 16, D-35392 Giessen, Germany

${ }^{28}$ KVI-CART, University of Groningen, NL-9747 AA Groningen, The Netherlands

${ }^{29}$ Lanzhou University, Lanzhou 730000, China

30 Liaoning University, Shenyang 110036, China

31 Nanjing Normal University, Nanjing 210023, China

32 Nanjing University, Nanjing 210093, China

33 Nankai University, Tianjin 300071, China

34 Peking University, Beijing 100871, China

35 Seoul National University, Seoul, 151-747 Korea

36 Shandong University, Jinan 250100, China

37 Shanghai Jiao Tong University, Shanghai 200240, China

38 Shanxi University, Taiyuan 030006, China

39 Sichuan University, Chengdu 610064, China

40 Soochow University, Suzhou 215006, China

41 Southeast University, Nanjing 211100, China

42 State Key Laboratory of Particle Detection and Electronics, Beijing 100049, Hefei 230026, China

43 Sun Yat-Sen University, Guangzhou 510275, China

44 Tsinghua University, Beijing 100084, China

45 (A)Ankara University, 06100 Tandogan, Ankara, Turkey; (B)Istanbul Bilgi University, 34060 Eyup, Istanbul, Turkey; (C)Uludag

University, 16059 Bursa, Turkey; (D)Near East University, Nicosia, North Cyprus, Mersin 10, Turkey

46 University of Chinese Academy of Sciences, Beijing 100049, China

47 University of Hawaii, Honolulu, Hawaii 96822, USA

48 University of Jinan, Jinan 250022, China

49 University of Minnesota, Minneapolis, Minnesota 55455, USA

${ }^{50}$ University of Muenster, Wilhelm-Klemm-Str. 9, 48149 Muenster, Germany

51 University of Science and Technology Liaoning, Anshan 114051, China

52 University of Science and Technology of China, Hefei 230026, China

53 University of South China, Hengyang 421001, China

${ }^{54}$ University of the Punjab, Lahore-54590, Pakistan

55 (A)University of Turin, I-10125, Turin, Italy; (B)University of Eastern Piedmont, I-15121, Alessandria, Italy; (C)INFN, I-10125, Turin, Italy

${ }^{56}$ Uppsala University, Box 516, SE-75120 Uppsala, Sweden

57 Wuhan University, Wuhan 430072, China

58 Xinyang Normal University, Xinyang 464000, China

59 Zhejiang University, Hangzhou 310027, China

60 Zhengzhou University, Zhengzhou 450001, China

${ }^{a}$ Also at Bogazici University, 34342 Istanbul, Turkey

${ }^{b}$ Also at the Moscow Institute of Physics and Technology, Moscow 141700, Russia

${ }^{c}$ Also at the Functional Electronics Laboratory, Tomsk State University, Tomsk, 634050, Russia

${ }^{d}$ Also at the Novosibirsk State University, Novosibirsk, 630090, Russia

$e$ Also at the NRC "Kurchatov Institute", PNPI, 188300, Gatchina, Russia

$f$ Also at Istanbul Arel University, 34295 Istanbul, Turkey

$g$ Also at Goethe University Frankfurt, 60323 Frankfurt am Main, Germany

${ }^{h}$ Also at Key Laboratory for Particle Physics, Astrophysics and Cosmology, Ministry of Education; Shanghai Key Laboratory for

Particle Physics and Cosmology; Institute of Nuclear and Particle Physics, Shanghai 200240, China

${ }^{i}$ Also at Government College Women University, Sialkot - 51310. Punjab, Pakistan

${ }^{j}$ Currently at: Center for Underground Physics, Institute for Basic Science, Daejeon 34126, Korea

Abstract: To investigate the nature of the $\psi(3770)$ resonance and to measure the cross section for $\mathrm{e}^{+} \mathrm{e}^{-} \rightarrow \mathrm{DD}, \mathrm{a}$ cross-section scan data sample, distributed among 41 center-of-mass energy points from 3.73 to $3.89 \mathrm{GeV}$, was taken with the BESIII detector operated at the BEPCII collider in the year 2010. By analyzing the large angle Bhabha scattering events, we measure the integrated luminosity of the data sample at each center-of-mass energy point. The total integrated luminosity of the data sample is $76.16 \pm 0.04 \pm 0.61 \mathrm{pb}^{-1}$, where the first uncertainty is statistical and the second systematic.

Keywords: Bhabha scattering events, integrated luminosity, BESIII 


\section{PACS: 13.66.De, 13.66.Jn～DOI: 10.1088/1674-1137/42/6/063001}

\section{Introduction}

The $\psi(3770)$ is the lowest mass charmonium state above the $\mathrm{D} \overline{\mathrm{D}}$ threshold, and is generally regarded as the $1^{3} \mathrm{D}_{1}$ dominant charmonium state [1]. To investigate the nature of the $\psi(3770)$ resonance, the BESIII Collaboration performed a cross-section scan experiment, in which $\mathrm{e}^{+} \mathrm{e}^{-}$data at 41 center-of-mass $(\mathrm{CM})$ energy $\left(E_{\mathrm{cm}}\right)$ points from 3.73 to $3.89 \mathrm{GeV}$ were collected. This data sample, referred to as the " $\psi(3770)$ cross-section scan data," was collected during the time period from June 1st to June 16th, 2010.

The $\psi(3770)$ cross-section scan data can be used to study the line-shapes of the cross sections for various hadronic final states produced in $\mathrm{e}^{+} \mathrm{e}^{-}$annihilation in the energy region around the $\psi(3770)$. Amplitude analyses of these line-shapes of cross sections will provide crucial information to explore the anomalous line-shape observed by the BESII experiment in 2008 [2]. These also benefit the measurements of the parameters of the $\psi(3770)$ resonance and shed light on the understanding of the branching fraction of $\psi(3770) \rightarrow$ non-DD $[3-7]$ decays.

In this paper, we present measurements of the integrated luminosity of the $\psi(3770)$ cross-section scan data at each $E_{\mathrm{cm}}$ by analyzing large angle Bhabha scattering events. We follow a method similar to that used in the measurement of the integrated luminosity of the data taken at $E_{\mathrm{cm}}=3.773 \mathrm{GeV}$ with the BESIII detector [8]. Furthermore, the luminosities are checked with an independent measurement by analyzing $\mathrm{e}^{+} \mathrm{e}^{-} \rightarrow(\gamma) \gamma \gamma$ events.

\section{BESIII detector}

BEPCII [9] is a double-ring $\mathrm{e}^{+} \mathrm{e}^{-}$collider. The design peak luminosity is $1 \times 10^{33} \mathrm{~cm}^{-2} \mathrm{~s}^{-1}$ at a beam current of $0.93 \mathrm{~A}$ and was achieved in 2016. The BESIII detector [9] has a geometrical acceptance of $93 \%$ of $4 \pi$ and consists of the following main components: 1) a smallcelled, helium-based main drift chamber (MDC) with 43 layers. The average single wire resolution is $135 \mu \mathrm{m}$, and the momentum resolution for $1 \mathrm{GeV} / c$ charged particles in a $1 \mathrm{~T}$ magnetic field is $0.5 \% ; 2$ ) an electromagnetic calorimeter (EMC) made of $6240 \mathrm{CsI}(\mathrm{Tl})$ crystals arranged in a cylindrical shape (barrel) plus two endcaps. For $1.0 \mathrm{GeV}$ photons, the energy resolution is $2.5 \%(5 \%)$ in the barrel (endcaps), and the position resolution is 6 $\mathrm{mm}$ (9 mm) in the barrel (endcaps); 3) a Time-Of-Flight system (TOF) for particle identification composed of a barrel part made of two layers with 88 pieces of $5 \mathrm{~cm}$ thick, $2.4 \mathrm{~m}$ long plastic scintillators in each layer, and two endcaps with 96 fan-shaped, $5 \mathrm{~cm}$ thick, plastic scin- tillators in each endcap. The time resolution is $80 \mathrm{ps}(110$ ps) in the barrel (endcaps), corresponding to a $2 \sigma \mathrm{K} / \pi$ separation for momentum up to about $1.0 \mathrm{GeV} / c$; 4) a muon chamber system (MUC) made of $1600 \mathrm{~m}^{2}$ of Resistive Plate Chambers (RPC) arranged in 9 layers in the barrel and 8 layers in the endcaps and incorporated in the return iron of the superconducting magnet. The position resolution is about $2 \mathrm{~cm}$.

\section{Method}

In principle, any process with a well-known crosssection can be used to determine the integrated luminosity of the corresponding data set. The luminosity $\mathcal{L}$ can be calculated by

$$
\mathcal{L}=\frac{N^{\mathrm{obs}} \times(1-\eta)}{\sigma \times \varepsilon},
$$

where $N^{\text {obs }}$ is the number of observed events, $\eta$ is the background contamination rate, $\sigma$ is the cross section and $\varepsilon$ is the detection efficiency.

In $\mathrm{e}^{+} \mathrm{e}^{-}$experiments, useful processes for the determination of integrated luminosity are the QED processes $\mathrm{e}^{+} \mathrm{e}^{-} \rightarrow(\gamma) \mathrm{e}^{+} \mathrm{e}^{-}, \mathrm{e}^{+} \mathrm{e}^{-} \rightarrow(\gamma) \gamma \gamma$ and $\mathrm{e}^{+} \mathrm{e}^{-} \rightarrow(\gamma) \mu^{+} \mu^{-}$ since they have precisely calculated cross sections in QED and relatively simple and distinctive final states. According to its largest production cross section, the Bhabha scattering process $\left(\mathrm{e}^{+} \mathrm{e}^{-} \rightarrow(\gamma) \mathrm{e}^{+} \mathrm{e}^{-}\right)$is used to measure the integrated luminosity of the $\psi(3770)$ crosssection scan data. In this work, Babayaga v3.5 [10] is adopted as the generator to determine the cross sections and the detection efficiencies.

\section{Luminosity measurement}

\subsection{Event selection}

The Bhabha scattering candidate events are selected by requiring exactly two oppositely-charged tracks that are well reconstructed in the MDC and satisfy $|\cos \theta|<$ 0.70 , where $\theta$ is the polar angle of the charged track. Each good charged track must satisfy $\left|V_{r}\right|<1 \mathrm{~cm}$ and $\left|V_{z}\right|<5 \mathrm{~cm}$. Here $V_{r}$ and $V_{z}$ are the closest distance of the charged tracks to the interaction point in the plane perpendicular to the beam direction and along the beam direction, respectively.

To suppress the backgrounds from $\mathrm{e}^{+} \mathrm{e}^{-} \rightarrow \mathrm{J} / \psi \mathrm{X}$, where the $\mathrm{J} / \psi$ decays into a $\mathrm{e}^{+} \mathrm{e}^{-}$pair, and $\mathrm{X}$ refers to $\gamma_{\mathrm{ISR}}, \pi^{0} \pi^{0}, \eta, \pi^{0}$, or $\gamma \gamma$, the sum of the momenta of the two good charged tracks is required to be greater than $0.9 \times E_{\mathrm{cm}} / c$. The momentum of each good charged track is also required to be less than $\left(E_{\mathrm{b}} / c+0.15\right) \mathrm{GeV} / c$, where $E_{\mathrm{b}}$ is the beam energy and $0.15 \mathrm{GeV} / c$ is about 
4 times the momentum resolution [8]. The energy deposited in the EMC of each charged track $\left(E_{\mathrm{EMC}}\right)$ is required to be larger than $1 \mathrm{GeV}$ to reject the background from $\mathrm{e}^{+} \mathrm{e}^{-} \rightarrow(\gamma) \mu^{+} \mu^{-}$.

After applying the above selection criteria, most of the surviving events come from the process $\mathrm{e}^{+} \mathrm{e}^{-} \rightarrow$ $(\gamma) \mathrm{e}^{+} \mathrm{e}^{-}$. Taking $E_{\mathrm{cm}}=3.7358 \mathrm{GeV}$ as an example, comparisons of the distributions of the momentum, polar angle and deposited energy in the EMC of the charged tracks between data and Monte Carlo (MC) simulation are shown in Fig. 1. Good agreement between data and MC simulation is observed.
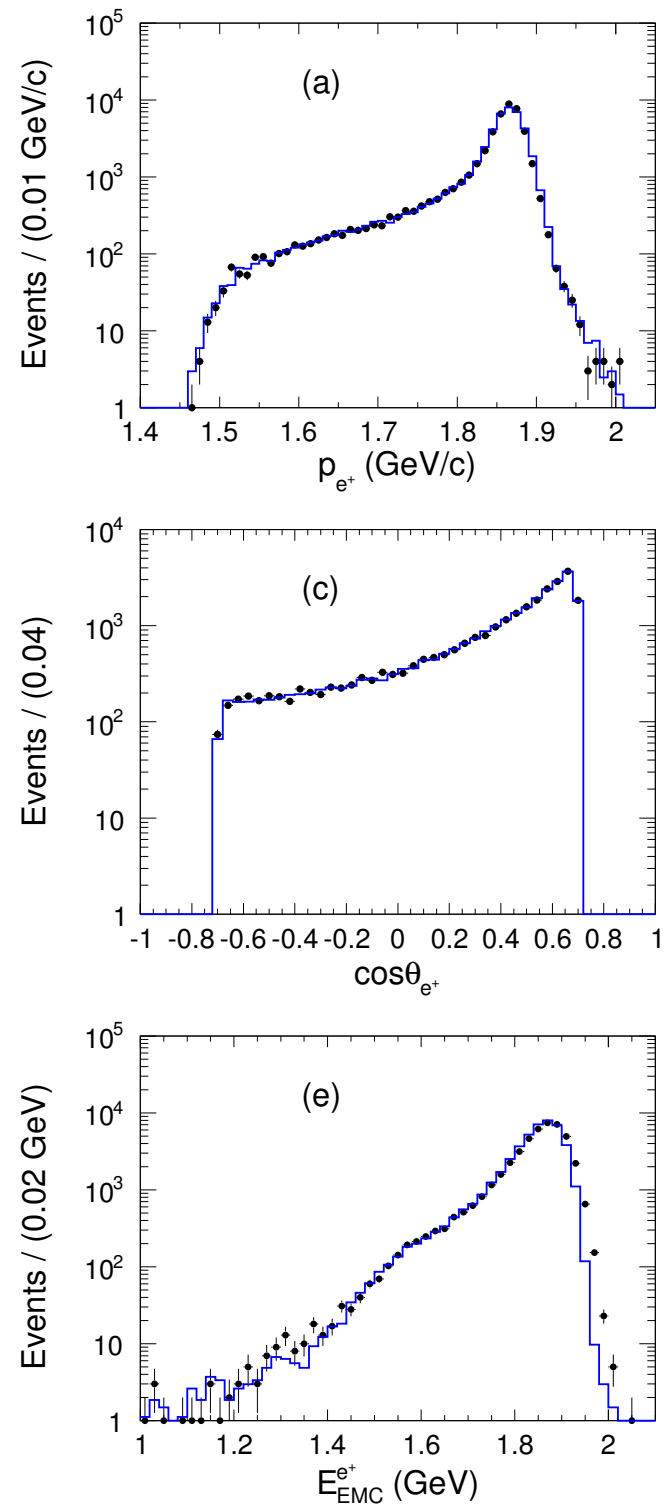

\subsection{Background estimation}

Most of the surviving candidate events are from $\mathrm{e}^{+} \mathrm{e}^{-} \rightarrow(\gamma) \mathrm{e}^{+} \mathrm{e}^{-}$. Potential background contamination includes two parts. One is the beam-associated background, such as beam-gas and beam-wall events. The other is background from $\mathrm{e}^{+} \mathrm{e}^{-}$reaction includ-

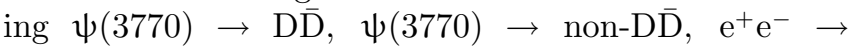
$(\gamma) \mathrm{J} / \psi,(\gamma) \psi(3686), \mathrm{q} \overline{\mathrm{q}},(\gamma) \mu^{+} \mu^{-}$and $(\gamma) \tau^{+} \tau^{-}$. To study the beam-associated backgrounds, we analyzed the separated-beam data samples collected at $3.400 \mathrm{GeV}$ and
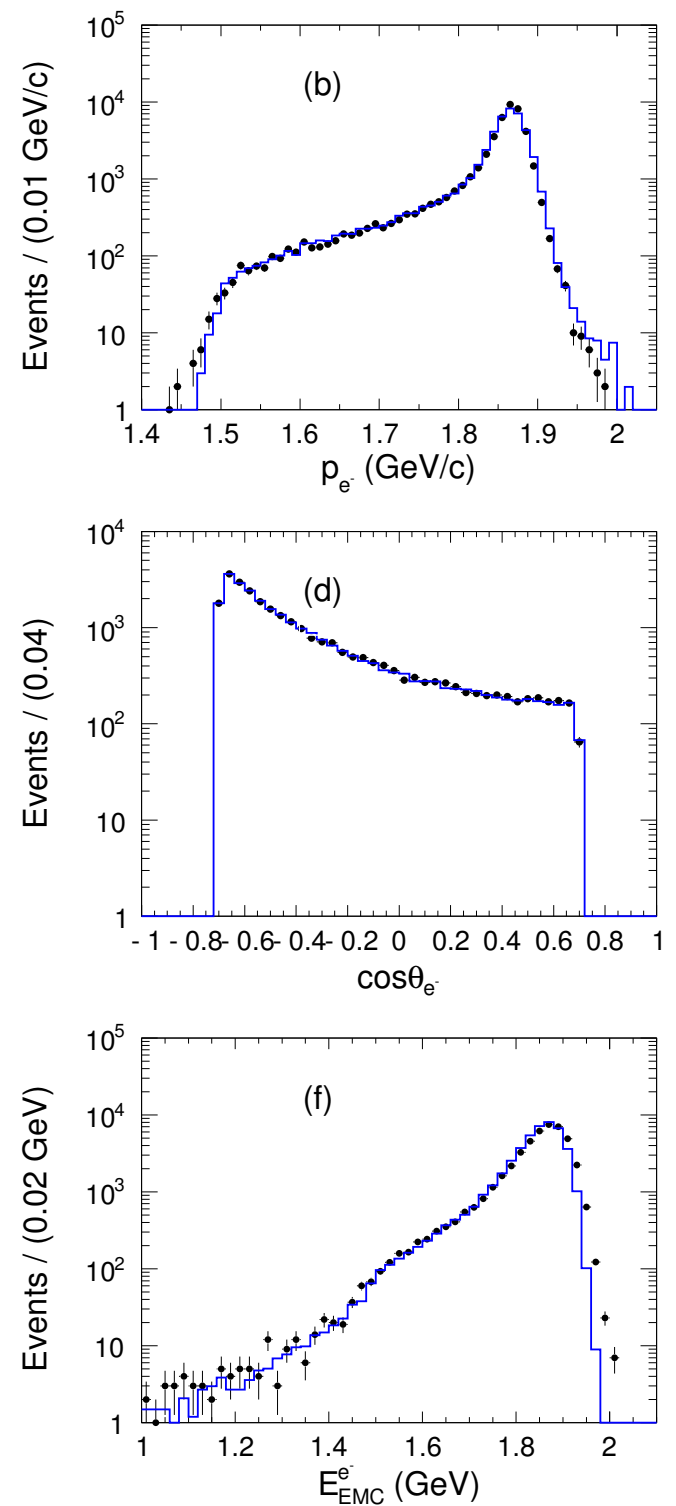

Fig. 1. (color online) Distributions of (a), (b) momentum, (c), (d) $\cos \theta$ and (e), (f) deposited energy in the EMC of the two charged tracks in the CM frame for selected Bhabha candidate events from the data taken at $E_{\mathrm{cm}}=3.7358$ $\mathrm{GeV}$ (points with error bars) and the corresponding MC simulation (histograms). The MC entries are normalized to the experimental data. 
Table 1. Summary of integrated luminosities measured using the processes $\mathrm{e}^{+} \mathrm{e}^{-} \rightarrow(\gamma) \mathrm{e}^{+} \mathrm{e}^{-}$ $\left(\mathcal{L}^{\mathrm{e}^{+} \mathrm{e}^{-}}\right)$and $\mathrm{e}^{+} \mathrm{e}^{-} \rightarrow(\gamma) \gamma \gamma\left(\mathcal{L}^{\gamma \gamma}\right)$ at each individual $\mathrm{CM}$ energy, where the first uncertainties are statistical and the second are systematic.

\begin{tabular}{ccc}
\hline$E_{\mathrm{cm}} / \mathrm{GeV}$ & $\mathcal{L}^{\mathrm{e}^{+} \mathrm{e}^{-} /\left(\mathrm{nb}^{-1}\right)}$ & $\mathcal{L}^{\gamma \gamma} /(\mathrm{nb}-1)$ \\
\hline 3.6471 & $2255.4 \pm 6.3 \pm 18.0$ & $2250.3 \pm 15.5 \pm 24.8$ \\
3.6531 & $2214.0 \pm 6.3 \pm 17.7$ & $2184.1 \pm 15.3 \pm 24.0$ \\
3.7266 & $896.2 \pm 4.1 \pm 7.2$ & $879.8 \pm 9.9 \pm 9.7$ \\
3.7356 & $334.8 \pm 2.5 \pm 2.7$ & $340.9 \pm 6.2 \pm 3.7$ \\
3.7358 & $491.9 \pm 3.0 \pm 3.9$ & $484.8 \pm 7.4 \pm 5.3$ \\
3.7376 & $327.7 \pm 2.5 \pm 2.6$ & $324.1 \pm 6.0 \pm 3.6$ \\
3.7447 & $956.0 \pm 4.2 \pm 7.6$ & $933.9 \pm 10.3 \pm 10.3$ \\
3.7464 & $1412.2 \pm 5.1 \pm 11.3$ & $1404.1 \pm 12.6 \pm 15.4$ \\
3.7488 & $2270.9 \pm 6.5 \pm 18.2$ & $2267.6 \pm 16.0 \pm 24.9$ \\
3.7503 & $2971.8 \pm 7.5 \pm 23.8$ & $2962.7 \pm 18.3 \pm 32.6$ \\
3.7526 & $3310.7 \pm 7.9 \pm 26.5$ & $3308.1 \pm 19.4 \pm 36.4$ \\
3.7541 & $3418.1 \pm 8.0 \pm 27.3$ & $3370.0 \pm 19.6 \pm 37.1$ \\
3.7555 & $3878.0 \pm 8.5 \pm 31.0$ & $3824.9 \pm 20.9 \pm 42.1$ \\
3.7585 & $4444.8 \pm 9.2 \pm 35.6$ & $4411.9 \pm 22.4 \pm 48.5$ \\
3.7616 & $4494.7 \pm 9.2 \pm 36.0$ & $4456.9 \pm 22.5 \pm 49.0$ \\
3.7645 & $3290.3 \pm 7.9 \pm 26.3$ & $3277.4 \pm 19.3 \pm 36.1$ \\
3.7675 & $2449.9 \pm 6.8 \pm 19.6$ & $2419.2 \pm 16.6 \pm 26.6$ \\
3.7705 & $2021.7 \pm 6.2 \pm 16.2$ & $2001.7 \pm 15.1 \pm 22.0$ \\
3.7735 & $1833.0 \pm 5.9 \pm 14.7$ & $1818.0 \pm 14.4 \pm 20.0$ \\
3.7765 & $1829.4 \pm 5.9 \pm 14.6$ & $1823.1 \pm 14.5 \pm 20.1$ \\
3.7795 & $1956.1 \pm 6.1 \pm 15.6$ & $1933.1 \pm 14.9 \pm 21.3$ \\
3.7825 & $2148.3 \pm 6.4 \pm 17.2$ & $2116.8 \pm 15.6 \pm 23.3$ \\
3.7855 & $2546.7 \pm 7.0 \pm 20.4$ & $2538.0 \pm 17.1 \pm 27.9$ \\
3.7882 & $2840.9 \pm 7.4 \pm 22.7$ & $2811.2 \pm 18.0 \pm 30.9$ \\
3.7925 & $3537.2 \pm 8.2 \pm 28.3$ & $3506.3 \pm 20.1 \pm 38.6$ \\
3.7964 & $4056.9 \pm 8.8 \pm 32.5$ & $4006.1 \pm 21.6 \pm 44.1$ \\
3.8002 & $3931.2 \pm 8.7 \pm 31.4$ & $3911.1 \pm 21.3 \pm 43.0$ \\
3.8026 & $2690.5 \pm 7.2 \pm 21.5$ & $2671.3 \pm 17.6 \pm 29.4$ \\
3.8064 & $1762.4 \pm 5.8 \pm 14.1$ & $1732.0 \pm 14.2 \pm 19.1$ \\
3.8095 & $1252.3 \pm 4.9 \pm 10.0$ & $1275.1 \pm 12.2 \pm 14.0$ \\
3.8124 & $898.5 \pm 4.2 \pm 7.2$ & $898.5 \pm 10.3 \pm 9.9$ \\
3.8156 & $683.0 \pm 3.6 \pm 5.5$ & $666.6 \pm 8.8 \pm 7.3$ \\
3.8236 & $399.5 \pm 2.8 \pm 3.2$ & $386.3 \pm 6.7 \pm 4.2$ \\
3.8315 & $281.7 \pm 2.3 \pm 2.3$ & $278.5 \pm 5.7 \pm 3.1$ \\
3.8396 & $282.3 \pm 2.4 \pm 2.3$ & $269.6 \pm 5.7 \pm 3.0$ \\
3.8475 & $279.8 \pm 2.4 \pm 2.2$ & $273.8 \pm 5.7 \pm 3.0$ \\
3.8557 & $318.8 \pm 2.5 \pm 2.6$ & $317.8 \pm 6.2 \pm 3.5$ \\
3.8636 & $302.3 \pm 2.5 \pm 2.4$ & $300.6 \pm 6.0 \pm 3.3$ \\
3.8715 & $514.2 \pm 3.2 \pm 4.1$ & $507.7 \pm 7.8 \pm 5.6$ \\
3.8805 & $190.1 \pm 2.0 \pm 1.5$ & $172.2 \pm 4.6 \pm 1.9$ \\
3.8905 & $184.1 \pm 1.9 \pm 1.5$ & \\
\hline & &
\end{tabular}

4.030 GeV with BESIII. To estimate the background contamination rates for the other background processes, we analyze large $\mathrm{MC}$ samples generated at $E_{\mathrm{cm}}=3.773 \mathrm{GeV}$. The overall contamination rate $\eta$ is estimated by

$$
\eta=\frac{\sum \sigma^{i} \times \eta^{i}}{\sigma^{\text {Bhabha }} \times \varepsilon^{\text {Bhabha }}}
$$

where $\sigma^{i}$ and $\eta^{i}$ are the cross section and the contamination rate for a specific process $i$, respectively; and $\sigma^{\text {Bhabha }}$ and $\varepsilon^{\text {Bhabha }}$ are the cross section and detection efficiency, respectively, for the Bhabha scattering process. The overall contamination rate of these backgrounds is estimated to be at the level of $10^{-4}$.

\subsection{Numerical result}

Inserting the numbers of observed Bhabha scattering events, the contamination rates of backgrounds, the detection efficiencies and cross sections calculated with the Babayaga v3.5 generator [10] into Eq. (1), we obtain the integrated luminosity at individual CM energy points for the $\psi(3770)$ cross-section scan data.

The measured integrated luminosities are summarized in the second column of Table 1 . The total integrated luminosity of the $\psi(3770)$ cross-section scan data is determined to be $76.16 \pm 0.04 \pm 0.61 \mathrm{pb}^{-1}$, where the first uncertainty is statistical and the second systematic, which will be discussed in the following.

\section{$5 \quad$ Systematic uncertainty}

The main sources of the systematic uncertainty are the event selection, the trigger efficiency, the generator, and the beam energy. Due to the low luminosity of individual data sets, we take the average value among the $41 \mathrm{CM}$ energy points as the systematic uncertainties to avoid large statistical fluctuations.

To estimate the systematic uncertainty of the $\cos \theta$ requirement, we repeat the measurements with the alternative requirements $|\cos \theta|<0.60,|\cos \theta|<0.65,|\cos \theta|<$ 0.75 , or $|\cos \theta|<0.80$, individually. The maximum relative change of the total integrated luminosity with respect to the nominal value is taken as the systematic uncertainty.

To study the systematic uncertainty arising from the MDC information, including the tracking and momentum requirements, we select a Bhabha sample using only EMC information. Two clusters must be reconstructed in the EMC with a deposited energy larger than $0.85 \times E_{\mathrm{b}}$ and a polar angle within $|\cos \theta|<0.7$. To remove $\mathrm{e}^{+} \mathrm{e}^{-} \rightarrow(\gamma) \gamma \gamma$ events, an additional requirement of $5^{\circ}<|\Delta \phi|<22^{\circ}$ is imposed, where $\Delta \phi$ is defined as $\Delta \phi=\left|\phi_{1}-\phi_{2}\right|-180^{\circ}$, and $\phi_{1}$ and $\phi_{2}$ are the azimuthal angles of the two showers in the EMC. The requirements on the MDC information are then imposed on the selected candidates, and the ratio of the surviving events is regarded as the corresponding acceptance efficiency. The difference of the acceptance efficiencies between data and MC simulation is taken as the relevant systematic uncertainty.

To estimate the systematic uncertainties of the EMC cluster reconstruction and $E_{\mathrm{EMC}}$ requirement, we select a Bhabha sample with almost the same selection requirements as those listed in Section 4.1 except for the de- 
posited energy requirement. Additional requirements of $E_{\mathrm{EMC}}>1.0 \mathrm{GeV}$ and $E_{\mathrm{EMC}} / p>0.8$ are imposed on one charged track and the other charged track is kept as the control sample. The difference of the acceptance efficiencies of the EMC cluster reconstruction and $E_{\mathrm{EMC}}$ requirement between data and $\mathrm{MC}$ simulation are taken as the systematic uncertainties.

The uncertainty of the trigger efficiency is less than $0.1 \%$ [11]. The systematic uncertainty due to background is negligible. The uncertainty associated with the signal MC model due to the Babayaga generator is assigned to be $0.5 \%$ according to Ref. [12]. To estimate the systematic uncertainty due to beam energy, we repeat the measurement by shifting the $\mathrm{CM}$ energies by $\pm 0.5, \pm 1$ or $\pm 2 \mathrm{MeV}$, individually. The largest change in total integrated luminosity with respect to the nominal value is assigned as the systematic uncertainty.

All of the systematic uncertainties are summarized in Table 2. Assuming the individual uncertainties to be independent, the total systematic uncertainty, $0.8 \%$, is calculated by adding them in quadrature.

Table 2. Summary of systematic uncertainties in the luminosity measurement using the processes $\mathrm{e}^{+} \mathrm{e}^{-} \rightarrow(\gamma) \mathrm{e}^{+} \mathrm{e}^{-}$and $\mathrm{e}^{+} \mathrm{e}^{-} \rightarrow(\gamma) \gamma \gamma$.

\begin{tabular}{ccc}
\hline source & \multicolumn{2}{c}{ systematic uncertainty $(\%)$} \\
\cline { 2 - 3 } $\mathrm{e}^{+} \mathrm{e}^{-} \rightarrow(\gamma) \mathrm{e}^{+} \mathrm{e}^{-}$ & $\mathrm{e}^{+} \mathrm{e}^{-} \rightarrow(\gamma) \gamma \gamma$ \\
\hline$|\cos \theta|<0.70$ & 0.2 & 0.2 \\
tracking and $p$ requirement & 0.5 & - \\
$E_{\mathrm{EMC}}$ requirement & 0.2 & 0.2 \\
EMC cluster reconstruction & 0.06 & 0.06 \\
$\Delta \phi$ requirement & - & 0.05 \\
trigger efficiency & 0.1 & 0.1 \\
generator & 0.5 & 1.0 \\
beam energy & 0.11 & 0.11 \\
\hline total & 0.8 & 1.1 \\
\hline
\end{tabular}

\section{Cross check}

As a cross check, we perform an independent measurement of the integrated luminosities of the $\psi(3770)$ cross-section scan data by analyzing the process $\mathrm{e}^{+} \mathrm{e}^{-} \rightarrow$ $(\gamma) \gamma \gamma$.

To select events from the process $\mathrm{e}^{+} \mathrm{e}^{-} \rightarrow(\gamma) \gamma \gamma$, we require that the number of good charged tracks is zero. Two neutral clusters are required to be within the polar angle region $|\cos \theta|<0.7$ and the deposited energy of each cluster in the EMC should be larger than $0.4 \times E_{\mathrm{b}}$. Since the directions of photons are not affected by the magnetic field, the two photon candidates should be back-to-back, and are required to satisfy $|\Delta \phi|<2.5^{\circ}$, where $\Delta \phi$ is defined as previously. Figure 2 shows a comparison of the $\Delta \phi$ distribution of the $\mathrm{e}^{+} \mathrm{e}^{-} \rightarrow(\gamma) \gamma \gamma$ candidate events between the data taken at $E_{\mathrm{cm}}=3.7358 \mathrm{GeV}$ and the corresponding MC simulation. Good agreement is visible.

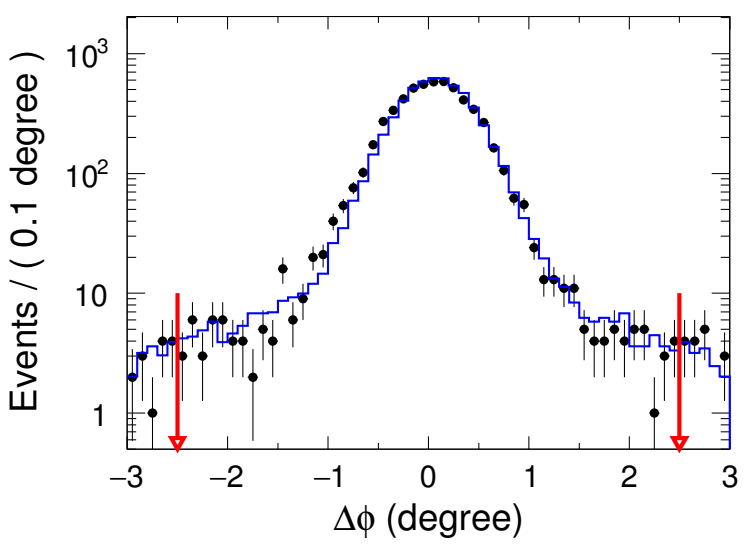

Fig. 2. (color online) The $\Delta \phi$ distributions of the $\mathrm{e}^{+} \mathrm{e}^{-} \rightarrow(\gamma) \gamma \gamma$ candidate events selected from the data taken at $E_{\mathrm{cm}}=3.7358 \mathrm{GeV}$ (points with error bars) and the corresponding MC simulation (histogram). The selected $\Delta \phi$ range is indicated by the two arrows. The MC entries are normalized to the experimental data.

For the background estimation, we analyzed the separated-beam data samples collected at $3.400 \mathrm{GeV}$ and $4.030 \mathrm{GeV}$ with BESIII, as well as MC samples

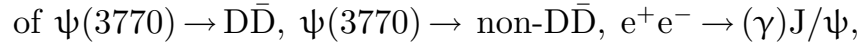
$(\gamma) \psi(3686), \mathrm{q} \overline{\mathrm{q}},(\gamma) \mathrm{e}^{+} \mathrm{e}^{-},(\gamma) \mu^{+} \mu^{-}$, and $(\gamma) \tau^{+} \tau^{-}$. The total contamination rate is estimated to be at the level of $10^{-3}$.

The integrated luminosity for the individual CM energy points is determined with Eq. (1) by using the numbers of observed $\mathrm{e}^{+} \mathrm{e}^{-} \rightarrow(\gamma) \gamma \gamma$ events, the contamination rates of backgrounds, the corresponding detection efficiencies, and cross sections calculated with the Babayaga v3.5 generator [10], as summarized in the third column of Table 1. The main sources of the systematic uncertainty arise from the EMC cluster reconstruction, the requirements on $|\cos \theta|, E_{\mathrm{EMC}}$ and $\Delta \phi$, the trigger efficiency, the generator, and the beam energy. Most sources are the same as those in the luminosity measurement using Bhabha scattering events, and the corresponding systematic uncertainties are determined with the same approach. To estimate the systematic uncertainty originating from the requirement on $\Delta \phi$, which is only used in the selection of $\mathrm{e}^{+} \mathrm{e}^{-} \rightarrow(\gamma) \gamma \gamma$ events, we repeat the measurements with the alternative requirements $|\Delta \phi|<2^{\circ}$ or $|\Delta \phi|<3^{\circ}$, individually. The maximum relative change of the integrated luminosity with respect to the nominal value is taken as the systematic uncertainty. The individual uncertainties are summarized in Table 2, and the total systematic uncertainty, $1.1 \%$, is obtained by assuming the different systematic sources independently 
and adding the individual values in quadrature. The total integrated luminosity measured using $\mathrm{e}^{+} \mathrm{e}^{-} \rightarrow(\gamma) \gamma \gamma$ events is $75.50 \pm 0.09 \pm 0.83 \mathrm{pb}^{-1}$, which is consistent with the result obtained using $\mathrm{e}^{+} \mathrm{e}^{-} \rightarrow(\gamma) \mathrm{e}^{+} \mathrm{e}^{-}$within uncertainties, but with relatively larger statistical and systematical uncertainties.

\section{Summary}

By analyzing $\mathrm{e}^{+} \mathrm{e}^{-} \rightarrow(\gamma) \mathrm{e}^{+} \mathrm{e}^{-}$events, we measure the integrated luminosities of the $\psi(3770)$ cross-section scan data taken at $41 \mathrm{CM}$ energy points. The total integrated luminosity of the $\psi(3770)$ cross-section scan data is determined to be $76.16 \pm 0.04 \pm 0.61 \mathrm{pb}^{-1}$, where the first uncertainty is statistical and the second systematic. As a cross check, we also perform a measurement of the integrated luminosity for the $\psi(3770)$ cross-section scan data using $\mathrm{e}^{+} \mathrm{e}^{-} \rightarrow(\gamma) \gamma \gamma$ events. The results are consistent with that of the previous measurement, but with relatively larger uncertainty. The obtained integrated luminosities at the individual CM energy points are summarized in Table 1 . The results provide important information needed to measure the cross sections of exclusive or inclusive hadronic production in $\mathrm{e}^{+} \mathrm{e}^{-}$ annihilation and thus benefit the understanding of the anomalous line-shape of $\mathrm{e}^{+} \mathrm{e}^{-} \rightarrow$ inclusive hadrons observed at BESII, the nature of the $\psi(3770)$, and the origin of the large branching fraction of $\psi(3770) \rightarrow$ nonDD decays [2].

The BESIII collaboration thanks the staff of BEPCII and the computing center for their hard efforts.

\section{References}

1 Y. B. Ding et al, Phys. Rev. D, 51: 5064 (1995)

2 M. Ablikim et al (BES Collaboration), Phys. Rev. Lett., 101: 102004 (2008)

3 M. Ablikim et al (BES Collaboration), Phys. Rev. D, 76: 122002 (2007)

4 M. Ablikim et al (BES Collaboration), Phys. Lett. B, 659: 74 (2008)

5 M. Ablikim et al (BES Collaboration), Phys. Rev. Lett., 97: $121801(2006)$

6 M. Ablikim et al (BES Collaboration), Phys. Lett. B, 641: 145
(2006)

7 D. Besson et al (CLEO Collaboration), Phys. Rev. Lett., 104: $159901(2010)$

8 M. Ablikim et al (BESIII Collaboration), Chin. Phys. C, 37: 123001 (2013)

9 M. Ablikim et al (BESIII Collaboration), Nucl. Instrum. Meth. A, 614: 345 (2010)

10 G. Balossini et al, Nucl. Phys. B, 758: 227 (2006)

11 N. Berger et al, Chin. Phys. C, 34: 1779 (2010)

12 C. M. Carloni Calame et al, Nucl. Phys. Proc. Suppl., 131: 48 (2004); G. Balossini et al, Phys. Lett. B, 663: 209 (2008) 